\title{
ESTRATEGIA PARA LA MEJORA DE LA COMUNICACIÓN Y LA INTELIGENCIA EMOCIONAL EN UNA EMPRESA DE TELECOMUNICACIONES CNT EP TUNGURAHUA
}

\section{STRATEGY FOR THE IMPROVEMENT OF COMMUNICATION AND EMOTIONAL INTELLIGENCE IN A TELECOMMUNICATION COMPANY CNT EP TUNGURAHU}

\begin{abstract}
Osmany Pérez Barral, PhD. Doctor en Ciencias Económicas (Cuba). Docente investigador de la Pontificia Universidad Católica del Ecuador, Sede Ambato, Ecuador. operez@pucesa.edu.ec

Johana Patricia Aldás León, Mgs. Magíster en Administración de Empresas (Ecuador). Directora de Talento Humano de la Empresa de telecomunicaciones CNT EP TUNGURAHUA, Ecuador. ipaa 20@hotmail.com

Daniela Judith Bermúdez Santana, Mgs. Magíster en Administración de Empresas (Ecuador). Docente de la Universidad Técnica de Ambato, Ecuador. dj.bermudez@uta.edu.ec

Mónica del Rocío Alarcón Quinapanta, Mgs. Magíster en Dirección de Empresas (Ecuador). Docente de la Universidad Regional Autónoma de Los Andes, Ecuador. monyalarcon7@gmail.com
\end{abstract}

ARTíCULO DE INVESTIGACIÓN

Recibido: 5 de julio de 2017

Aceptado: 14 de agosto de 2017

ISSN: 1390-9320, Vol. 4, No.5, octubre 2017 


\section{RESUMEN}

La investigación propone una estrategia para la mejora de la comunicación y la inteligencia emocional en una empresa de telecomunicaciones: Corporación Nacional de Telecomunicaciones Empresa Pública (CNT EP) Tungurahua, el cual se inicia, con el estudio de las tendencias de comunicación y los procesos de inteligencia emocional que se administran con los trabajadores de una empresa de servicios de telecomunicaciones. Para alcanzar este objetivo, se diseña una estrategia que permita mejorar las relaciones internas de la Institución, utilizándose metodologías de estudio como: el análisis documental, el cuestionario y la entrevista; obteniendo información de los empleados, jefes y supervisores que trabajan en la CNT EP Tungurahua. Los resultados de la investigación orientan a desarrollar actividades correctivas, con el fin de lograr una comunicación efectiva y una inteligencia emocional coordinada entre los miembros que conforman la corporación, para lo cual es necesario la participación eficiente, eficaz y efectiva de los líderes y grupos estratégicos en todas las áreas funcionales, a fin de alcanzar el clima laboral esperado.

Palabras clave: comunicación; estrategia, inteligencia emocional

\section{ABSTRACT}

This research project proposes a strategy for the improvement of communication and emotional intelligence in a telecommunications company, CNT EP Tungurahua. It begins with the study of communication trends and the processes of emotional intelligence that are administered with the workers of a telecommunication services company. In order to meet this objective, a strategy is designed that makes it possible to improve the internal relations of the institution using study methodologies such as documentary analysis, questionnaire and interview and this information that is getting from the employees, bosses and supervisors who work at CNT EP Tungurahua National Corporation of Telecommunications. The research results help to develop corrective activities that will achieve effective communication and a coordinated emotional intelligence among the members of the corporation. For this reason, efficient and effective participation is need from the leaders and strategic groups in all of the functional areas in order to reach the anticipated work climate.

ISSN: 1390-9320, Vol. 4, No.5, octubre 2017 
Keywords: communication; strategy, emotional intelligence

\section{INTRODUCCIÓN}

Las empresas de telecomunicaciones en el Ecuador se basan en la generación de innovación, desarrollo y tecnología. Este perfil establece en la mente de los consumidores la necesidad de utilizar constantemente el servicio de información, al convertirse en un estatus global. En este sentido, las compañías de estas industrias son realmente competitivas y las encargadas de establecer capacidades de comunicación e inteligencia emocional en sus empleados, lo que motiva a la máxima dirección a investigar la efectividad de aplicar estrategias idóneas que generen un ambiente laboral favorable para el personal a su cargo. En este sentido, el estudio se convierte en el punto clave de desarrollo de una organización, al enfocar su atención a la gestión del talento humano, lo cual impulsa a convertir a los empleados en administrares de sus funciones con una comunicación fluida.

En la actualidad, las instituciones se encargan de llevar una administración controlada en su recurso humano, estableciéndose estrategias de participación, gestión del talento humano y clima laboral estable, con el objetivo de aplicar un equilibrio en la parte operativa y emocional del personal, creándose un enfoque de negociación y de inteligencia emocional. Asimismo, en la práctica empresarial, las organizaciones requieren simplificar el uso de sus recursos operativos y perfeccionar la comunicación y la inteligencia emocional de sus empleados, pues, al ser considerado un proceso complejo no se escatima su estudio y aplicación.

En tal sentido, las relaciones interpersonales deben adecuarse a la productividad y al cumplimiento de metas con la intervención de personas que ejecuten un concepto de comunicación e inteligencia emocional, con lo cual, se pone de manifiesto la importancia de aplicar una herramienta de solución a los posibles inconvenientes en los procesos de gestión de talento humano en empresas de telecomunicaciones. De esta manera, el objetivo general del estudio se basa en implementar una estrategia para la mejora de la comunicación y la inteligencia emocional en una Empresa de telecomunicaciones CNT EP TUNGURAHUA.

ISSN: 1390-9320, Vol. 4, No.5, octubre 2017 


\section{REVISIÓN TEÓRICA}

\section{Estado del Arte y la Práctica}

Los estudios enfocados a la comunicación y a la inteligencia emocional en los ámbitos de sociabilización humana, se materializan en el momento que se diseñan modelos para la mejora de las relaciones humanas, las ventajas que se adquieren de esta aplicación contribuyen a tener personas con éxito laboral, y son aquellas que tienen el $23 \%$ de las capacidades intelectuales y el $77 \%$ a las aptitudes emocionales (Peña, 2012). En el estudio realizado por Zárate (2012), se identifica que las personas tienen dos clases de inteligencias: la racional y la emocional, estas trabajan en equipo o asociadas para permitir administrar las emociones y enfocarse a una comunicación integral.

En la práctica, las relaciones interpersonales en el desarrollo de las actividades en una empresa, se identifican por la expresión en el manejo de la autoconciencia y la autorregulación, el mismo que se relaciona a la empatía y al trabajo en equipo entre colaboradores (Fienco e Itúrburo, 2011). Creándose, una filosofía de trabajo integradora, donde las decisiones que se toman son colectivas. Asimismo, en la teoría de Goleman (1995), se establecen estudios de la autoconciencia, la autorregulación, auto motivación, empatía y habilidades sociales, ya que crea ideas que contribuyen a ejercer un estudio desde una perspectiva analítica de las consecuencias psicológicas del comportamiento humano, acreditándose el desarrollo que surge desde su aplicación. De esta manera, se logra ser proactivo en el puesto de trabajo generando un servicio eficiente, eficaz y efectivo para el cliente externo.

La ejecución de una estrategia para la mejora de la comunicación y la inteligencia emocional tiene una importancia alta en la aplicación práctica, lo identifican los autores Bradberry \& Graves (2012) y Troya (2014), en sus investigaciones refiriéndose a las emociones y motivaciones como las acciones más relevantes en el proceso de interacción humana. En este sentido, el empleado debe sentirse motivado para brindar un servicio amable, que satisfaga las expectativas de los clientes externos, esto garantiza el incremento de nuevos demandantes del servicio en la empresa. De esta manera se entiende que los estudios realizados por Goleman (1995, 1999, 2002 y 2006), se orienten netamente al manejo de las 
cinco habilidades. Estas herramientas de estudio estabilizan este estudio y generan ideas que aporten a la elaboración de la estrategia, el cual contempla un abanico de posibilidades a ser utilizados en la aplicación de la estrategia para la comunicación y la inteligencia emocional. O sea, la comunicación y la inteligencia emocional son variables que deciden la calidad del servicio que brinda cualquier organización.

\section{MATERIALES Y MÉTODOS}

El análisis del presente estudio se guía en modelos determinados en este ámbito. La búsqueda de la estrategia para la mejora de la comunicación y la inteligencia emocional en una empresa de telecomunicaciones, se basa en los lineamientos laborales del personal que trabaja en la empresa de telecomunicaciones CNT EP Tungurahua, apoyado en los conceptos de autoconciencia, autorrealización, motivación, empatía y habilidades sociales.

Las empresas de la industria del servicio que se encuentran realizando cambios en sus estrategias para el manejo del talento humano, se enfocan en poseer personal especializado en el manejo de la comunicación y la inteligencia emocional, direccionada a obtener un ambiente laboral estratégico y creándose un rendimiento efectivo para la consecución de los objetivos. De esta manera, el estudio se orienta a la obtención de una estrategia idónea para los diferentes tipos de conducta humana y al diseño íntegro de herramientas de gestión para el proceso de cambio en los empleados.

Al respecto, las empresas de servicios en telecomunicaciones se caracterizan por poseer competencia, servicio, atención y calidad, ya que giran en el mismo entorno competitivo y se obligan a mantener la gestión de su talento humano bien definido y en las áreas que correspondan de su atención y ejecución. En este sentido, fue importante aplicar encuestas a los directivos y trabajadores de las diferentes áreas, con el objetivo de saber cómo desarrollan sus labores y los tipos de inteligencias que emplean para ejecutar sus funciones y atender adecuadamente a los clientes.

ISSN: 1390-9320, Vol. 4, No.5, octubre 2017 
El diagnóstico documental de los procesos, procedimientos y programas, fundamenta el direccionamiento, administración y control del talento humano de la organización para conocer la situación actual del personal, los mismos que se detallan a continuación:

1. Según el reglamento de gestión de talento humano vigente, direcciona los procesos del funcionamiento institucional, estableciendo, normas, mecanismos, técnicas y procedimientos, que permiten garantizar la administración del talento humano bajo preceptos de eficiencia y eficacia, buscándose la mayor productividad.

2. El procedimiento de comunicación, establece los lineamientos en los que se rige la empresa de telecomunicaciones CNT EP Tungurahua, como medios electrónicos internos, encuestas, entrevistas, entre otras.

3. La planificación de las capacitaciones en temas de motivación, comunicación, inteligencia emocional y gestión del cambio, son escasos y poco convencionales, la cual debe generarse según las necesidades de cada área funcional.

4. Existe un procedimiento y un flujograma de medición del clima laboral a nivel nacional.

5. El código de ética de la institución tiene el propósito de orientar la gestión, los principios, valores y políticas institucionales que regulan las relaciones internas y externas, de los servidores y servidoras de la empresa de telecomunicaciones CNT EP Tungurahua.

Según el análisis documental, el cual se enfoca en la medición de la situación actual del personal, un estudio predominante realizado en el año 2011, fue la medición del clima laboral, en el área comercial de la empresa de telecomunicaciones CNT EP Tungurahua, el cual arrojó un porcentaje promedio de $67 \%$ de cumplimiento sobre las variables de: inteligencia emocional, percepción del cliente interno, satisfacción laboral, relaciones interpersonales, productividad, clima laboral, cultura organizacional, ambiente físico y trabajo.

De acuerdo al estudio establecido en este ámbito, las autoridades y el área de talento humano realizan el seguimiento respectivo al cumplimiento de los indicadores mencionados en éste párrafo, sin embargo, no se ha obtenido los resultados esperados.

ISSN: 1390-9320, Vol. 4, No.5, octubre 2017 
Para obtener resultados en el método de estudio, se especifica en esta investigación la caracterización de las variables y los estilos de comportamientos mediante el método DISC $\circledast$, el mismo que muestra los estilos que posee cada individuo y que sirven para explorar e identificar el perfil adecuado de las relaciones. Para esto, se especifica primero las variables que se derivan de la comunicación y la inteligencia emocional, aspecto que se aborda en el siguiente epígrafe.

\section{Determinación de las variables que se derivan de la comunicación y la inteligencia emocional}

En este apartado, se presentan las variables que se derivan de la Comunicación y la Inteligencia Emocional a fin de conocerlas y establecer en base a ellas la estrategia a implementarse (Ver esquema 1).

Esquema 1. Variables que se derivan de la Comunicación

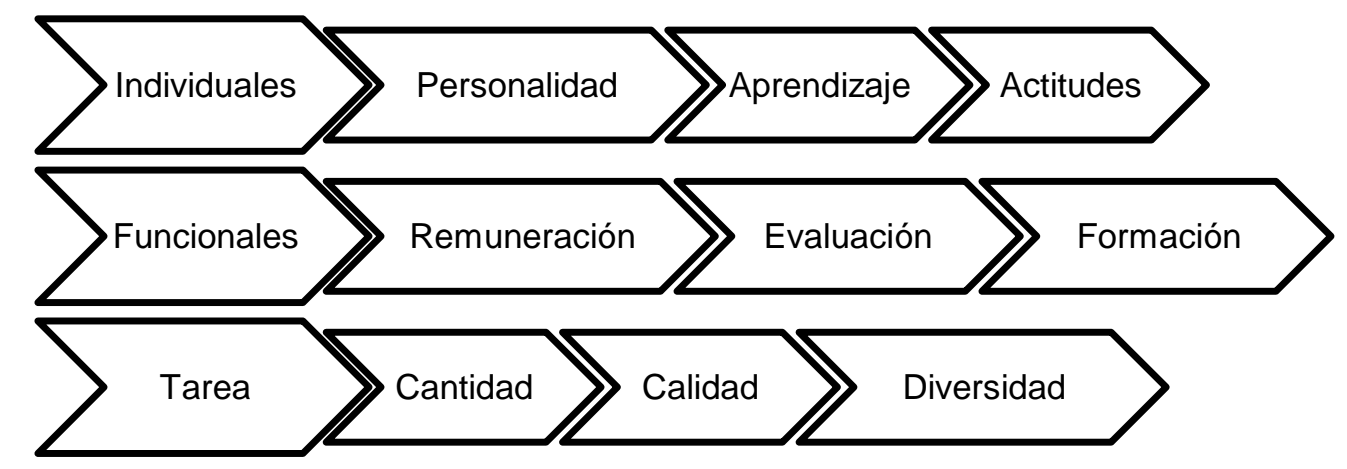

Fuente: elaboración propia a partir de Urbano (2012).

Las variables presentadas en la comunicación (Esquema 2), tienen un grado de afectación y percepción desde el punto de vista subjetivo en los individuos, pues se enmarcan en: cualidades personales, perspectivas de desarrollo y en cómo pueden ejecutar sus labores. Por ejemplo, el aprendizaje se caracteriza por la calidad de captación de la información, y; la actitud se materializa con el grado del comportamiento que proviene en la interacción con los clientes. 
Esquema 2. Variables que se derivan de la Inteligencia Emocional
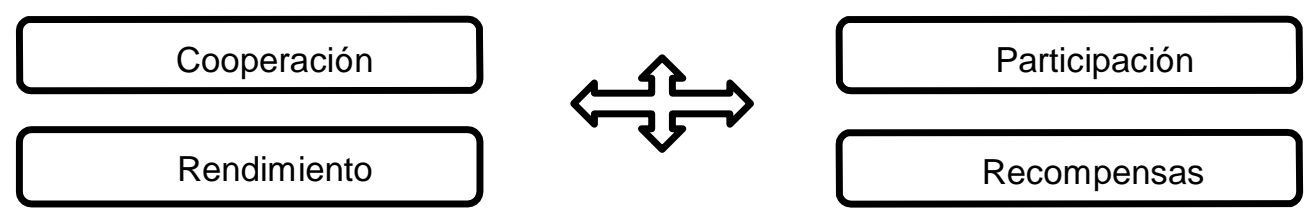

Fuente: elaboración propia a partir de Goleman (1999).

Las variables que se desglosan en la comunicación y la inteligencia emocional, especifican el comportamiento y/o conductas de las personas que trabajan de forma grupal e individual, las cuales se desarrollan mediante el conocimiento del método DISC® (dominante, influyente, estable y concienzudo), cada comportamiento identifica las diferencias humanas, comprendiéndose la diversidad existente en los estilos personales. Este método (Esquema 3), se considera integrador desde el punto de vista de las cualidades que deben tener las personas al momento de comunicarse partiendo de la inteligencia emocional que desarrollan.

En tal sentido se quiere destacar, que la inteligencia emocional debe aportar al desarrollo de las personas desde el punto de vista del trabajo en equipo, ser colaborativos y rendir al máximo en sus funciones, pues esto influye positivamente en la comunicación organizacional.

Esquema 3. Método DISC® (dominante, influyente, estable y concienzudo)

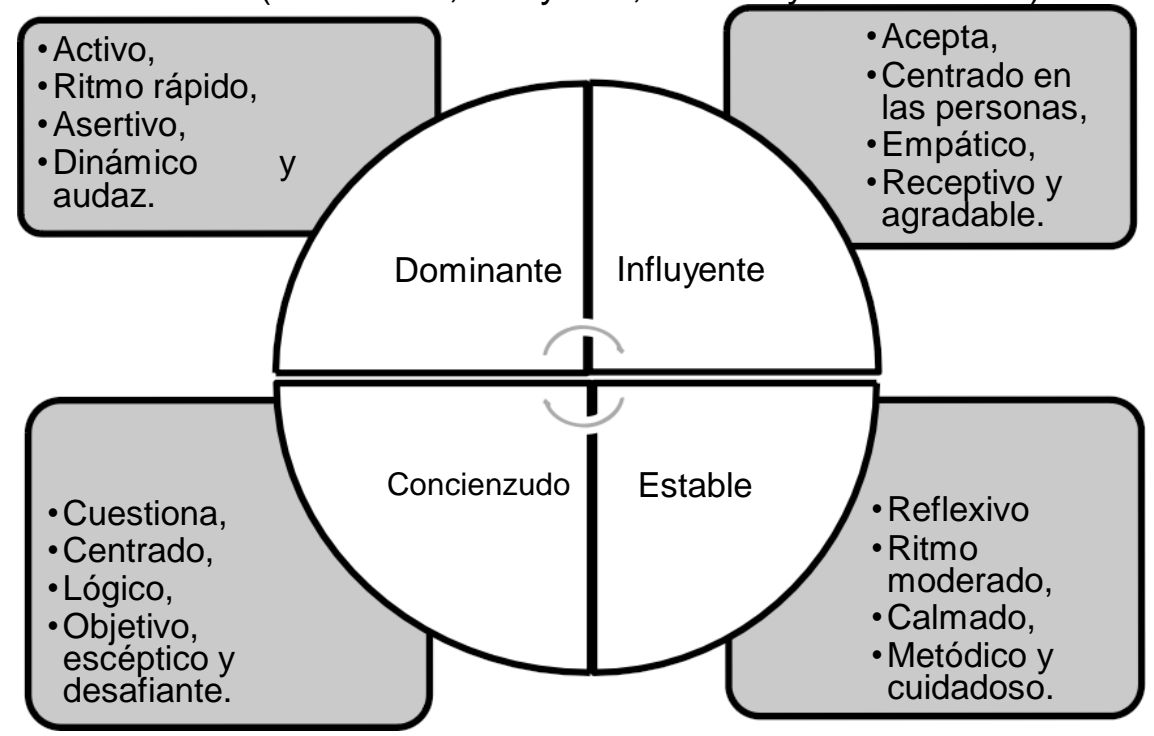

Fuente: tomado del Programa Cóndor aplicado a la CNT EP (2015). 
Los estilos que se manejan en el método DISC®, se caracterizan por los siguientes enfoques:

\section{D-Dominante}

- Énfasis: modificar su entorno, superándose la oposición y los desafíos.

- Tendencias: obtener resultados inmediatos, actuar y tomar medidas, aceptar desafíos.

- Motivado por: desafíos, poder y autoridad, respuestas directas.

- Teme: perder el control en su entorno, que se aprovechen de él/ella.

- Usted notará: auto-confianza, estilo directo, decidido y dispuesto/a tomar riesgos.

- Limitaciones: falta de interés o preocupación por los demás, impaciencia.

\section{I- Influyente}

- Énfasis: modificar su entorno, persuadiendo a los demás e influyendo sobre ellos.

- Tendencias: relaciones con personas y generar una impresión favorable.

- Motivado por: reconocimiento social, actividades grupales y relaciones.

- Teme: rechazo social, desaprobación y perder influencia.

- Usted notará: entusiasmo, encanto y sociabilidad.

- Limitaciones: impulsividad, desorganización, y poca capacidad para terminar lo iniciado.

\section{S-Estable}

- Énfasis: lograr estabilidad y completar tareas colaborando con otros.

- Tendencias: calmado, paciente, leal y sabe escuchar.

Motivado por: cambios infrecuentes, estabilidad, aprecio sincero y cooperación.

Teme: pérdida de estabilidad, lo desconocido, el cambio y lo impredecible.

- Usted notará: paciencia, tendencia a trabajar en equipo, estabilidad, enfoque sistemático y actitud calmada.

- Limitaciones: demasiado dispuesto a dar, poniéndose sus propias necesidades como últimas.

\section{C-Concienzudo}

Énfasis: trabajar dentro de las circunstancias para garantizar calidad y precisión.

- Tendencias: atención a estándares y detalles y pensamiento analítico.

- Motivado por: expectativas de desempeño claramente definidas, que la calidad y la precisión sea apreciada. 
- Teme: crítica su trabajo y métodos descuidados.

- Usted notará: comportamiento cauteloso, preciso, diplomático y controlado.

Limitaciones: demasiado crítico de sí mismo y de los demás, indecisión por un deseo de reunir, y analizar datos.

Las especificaciones en los comportamientos del método $\mathrm{DISC} \circledast$, permite explorar las preferencias de cada estilo, comprendiéndose las implicaciones, características y diversidad de cada persona.

\section{ANÁLISIS DE RESULTADOS Y DISCUSIÓN}

Una vez analizada la metodología de la investigación y al concretar los resultados que se reflejan en la encuesta y en la entrevista (instrumentos de investigación), los mismos que identifican a la comunicación y la inteligencia emocional como una necesidad irrelevante en la vida laboral de las empresas, se verifican las siguientes necesidades:

Cambiar el ambiente laboral de los empleados, realizándose capacitaciones de comunicación e inteligencia emocional.

Mejorar los planes de acción para mejorar el liderazgo y el trabajo en equipo. De esta manera se logra, aplicar nuevas técnicas en la relación interpersonal entre jefes, mandos medios y personal, donde prime el respeto mutuo y la capacidad de escucha en la toma de decisiones.

Realizar cambios internos para incentivar la comunicación efectiva entre áreas. Donde el personal de un área se interese por los resultados de las otras áreas de la organización. Esto se puede lograr, previa coordinación con los responsables de cada área para que se entregue información clara, precisa y en tiempo, en aras de no retrasar el resultado final de otras áreas, con el fin de compartir y hacer partícipe a todos de la información de la empresa.

Aplicar prácticas en el manejo de la administración del talento humano con los jefes y mandos medios, crear condiciones para la preparación de los cuadros de dirección de cada área. En este sentido, los jefes de área y supervisores deben identificar los diferentes tipos de personalidades, a fin de que se pueda orientar, encaminar y 
direccionar el alcance de los objetivos. De esta manera, se logra mejorar también el trato de los empleados y de los clientes externos.

Identificar los errores que se emiten desde los jefes y/o supervisores hacia sus subordinados y que se encuentran inconformes con la administración actual, a fin de realizar acciones correctivas y cambios según el Head Count de la Institución.

La empresa de telecomunicaciones CNT EP Tungurahua, necesita reforzar la comunicación mediante la aplicación de inducciones periódicas al personal para que los mismos conozcan la filosofía de trabajo de la organización, los procesos, procedimientos, normas, leyes, entre otros.

\section{Propuesta de Estrategia}

Una vez analizado el campo de las necesidades para mejorar la comunicación y la inteligencia emocional de la empresa de telecomunicaciones CNT EP Tungurahua, se materializa las acciones para generar una estrategia que contemple el desarrollo equilibrado de las relaciones y la comunicación entre el personal de la Institución, la misma que se estandariza de la siguiente manera:

Las estrategias que se desarrollan en este estudio se derivan de la aplicación de los objetivos del Alineamiento Estratégico de la empresa de telecomunicaciones CNT EP Tungurahua, la cual, se refleja en estrategias de innovación y transformación empresarial. Siendo las tres aristas predominantes en el enfoque empresarial, como: crecimiento, productividad y sostenibilidad; destacándose la mejora de la comunicación y la inteligencia emocional.

Las variables que se contemplan en este enfoque, instauran características idóneas que se requieren en la aplicación de un marco estratégico en la empresa telecomunicaciones CNT EP Tungurahua, basadas en la comunicación y la inteligencia emocional, creadas y descritas a continuación:

a) Operativa, se enlaza con la autorregulación en el ámbito empresarial, cronograma de horarios, y reuniones periódicas con el personal interno en cada área de trabajo, a fin 
de establecer compromisos específicos en el ámbito de comunicación e inteligencia emocional.

b) Táctica, se relaciona con la empatía, porque se enfoca en el fortalecimiento de alianzas estratégicas con todo el personal.

c) Estratégica, se enlaza con la motivación para cumplir con el reconocimiento, la participación y el cumplimiento de los planes de acción.

d) Horizontal, se integra con las habilidades sociales los jefes y mandos medios ejecutan las habilidades de liderazgo y promueven los cambios, y proponen las gestiones para alcanzar y solicitar los recursos que se requieren.

e) Informal, se interrelaciona con la autoconciencia, los jefes, mandos medios y subordinados concilian reuniones para llegar a acuerdos positivos, utilizándose el conocimiento en programación neurolingüística $(\mathrm{PNL})$, herramienta que permite la comunicación interpersonal combinándose el cerebro, el lenguaje y la experiencia.

En tal sentido, para el monitoreo del cumplimiento de los objetivos se toma en consideración la matriz cuantitativa de la planificación estratégica (MCPE), ésta determina los factores del atractivo relativo de las estrategias alternativas, a fin de elegir las variables que más atención requieren para su seguimiento y cumplimiento, la cual se detalla a continuación:

Cuadro 1. Cronograma de actividades para el cumplimiento de las estrategias

\begin{tabular}{|c|c|c|c|}
\hline Estrategia & Actividades & $\begin{array}{c}\text { Fecha de } \\
\text { cumplimiento }\end{array}$ & Responsables \\
\hline $\begin{array}{l}\text { Realizar alianzas } \\
\text { estratégicas con el } \\
\text { personal afín y } \\
\text { proactivo para que } \\
\text { se formen grupos } \\
\text { de liderazgo } \\
\text { organizacional. }\end{array}$ & $\begin{array}{l}\text {-Realizar reuniones para } \\
\text { nombrar democráticamente } \\
\text { una persona dinámica y con } \\
\text { dotes de liderazgo para que } \\
\text { coordine y conforme grupos } \\
\text { organizacionales r que } \\
\text { promuevan la comunicación y } \\
\text { la inteligencia emocional en } \\
\text { cada área. } \\
\text {-Ejecutar acciones que } \\
\text { permitan monitorear la } \\
\text { comunicación y la inteligencia } \\
\text { emocional del personal. }\end{array}$ & $\begin{array}{l}\text { Enero y } \\
\text { febrero } 2017\end{array}$ & $\begin{array}{l}\text { Jefes de área, } \\
\text { supervisores y } \\
\text { talento humano }\end{array}$ \\
\hline
\end{tabular}

ISSN: 1390-9320, Vol. 4, No.5, octubre 2017 


\begin{tabular}{|c|c|c|c|}
\hline & $\begin{array}{l}\text {-Identificar las falencias que } \\
\text { están ocasionando la } \\
\text { distorsión de la información y } \\
\text { las emociones, tomándose un } \\
\text { accionar inmediato. }\end{array}$ & $\begin{array}{l}\text { Marzo y abril } \\
2017\end{array}$ & \\
\hline $\begin{array}{lr}\text { Ejecutar cambios } \\
\text { temporales } & \text { y } \\
\text { dinámicos en los } \\
\text { puestos } & \text { de } \\
\text { personal según el } \\
\text { Head Count }\end{array}$ & $\begin{array}{l}\text { - Identificar a los colaboradores } \\
\text { que tienen problemas de } \\
\text { comunicación e inteligencia } \\
\text { emocional y reubicarlos } \\
\text { temporalmente para controlar } \\
\text { su desempeño en las otras } \\
\text { áreas. } \\
\text {-Diagnosticar los traslados } \\
\text { administrativos y verificar que } \\
\text { se esté cumpliendo con el } \\
\text { objetivo. } \\
\text {-Evaluar los cambios } \\
\text { realizados, informándose de } \\
\text { los pro y contra de los mismos. }\end{array}$ & $\begin{array}{l}\text { Mayo, junio y } \\
\text { julio } 2017\end{array}$ & $\begin{array}{l}\text { Área de } \\
\text { Talento } \\
\text { Humano }\end{array}$ \\
\hline $\begin{array}{l}\text { Establecer un plan } \\
\text { de acción para los } \\
\text { jefes de área y } \\
\text { mandos medios en } \\
\text { temas de } \\
\text { administración del } \\
\text { talento humano y } \\
\text { emociones }\end{array}$ & $\begin{array}{l}\text {-Verificar las ventajas y } \\
\text { desventajas del ambiente } \\
\text { laboral de cada área. } \\
\text {-Diagnosticar los problemas } \\
\text { que surgen en el liderazgo de } \\
\text { los jefes de áreas y } \\
\text { supervisores. } \\
\text {-Establecer capacitaciones } \\
\text { que permitan identificar la } \\
\text { debilidad en la administración } \\
\text { de la comunicación y la } \\
\text { inteligencia emocional de los } \\
\text { colaboradores que } \\
\text { encuentran bajo el cargo de } \\
\text { los jefes de área y } \\
\text { supervisores. }\end{array}$ & $\begin{array}{l}\text { Septiembre } \\
2017 \\
\text { Octubre } 2017\end{array}$ & $\begin{array}{l}\quad \text { Grupos de } \\
\text { trabajo, líderes } \\
\text { designados y } \\
\text { Talento } \\
\text { Humano }\end{array}$ \\
\hline
\end{tabular}

Fuente: elaboración propia. 


\section{CONCLUSIONES}

El estudio teórico coadyuvó al desarrollo de las estrategias para la empresa telecomunicaciones CNT EP Tungurahua, pudiéndose identificar las variables que influyen en la comunicación y la inteligencia emocional, destacándose la importancia que tienen para ofrecer un servicio de calidad al cliente.

Basado en el desarrollo metodológico de los autores, se reconoce la importancia que tiene la participación de los trabajadores de la organización en el proceso estratégico, pues son los que trabajan directamente con el cliente. En este sentido, la comunicación interna y externa debe ser precisa y proactiva para el buen funcionamiento de la organización con su personal y con el entorno, esto garantiza un grado de competitividad diferenciador con respecto a sus competidores.

El análisis de campo realizado a los colaboradores de la institución, permitió determinar la situación actual en el manejo de la comunicación y la inteligencia emocional, lográndose identificar las debilidades que éstas demandan. En este sentido, el análisis facilitó la construcción de estrategias que apalanquen a la correcta comunicación y el manejo de las emociones, destacándose un enfoque empresarial a partir del crecimiento organizacional y personal, de la productividad del trabajo y la sostenibilidad de la organización como resultado de la mejora de la comunicación y la inteligencia emocional.

Se identificaron las falencias en el ámbito comunicacional y de emociones, las mismas que necesitan el mejoramiento efectivo y el seguimiento continuo para el mejoramiento del clima laboral de las diferentes áreas de la organización. Estas falencias fueron atendidas al momento de proponer las estrategias a seguir en la empresa telecomunicaciones CNT EP Tungurahua, enfocadas a gestionar alianzas estratégicas, movilidad o promoción de puestos de trabajos según los resultados alcanzados y a la preparación de directivos y mandos intermedios en temas de talento humano y manejo de emociones.

\section{REFERENCIAS BIBLIOGRÁFICAS}

Bradberry, T., \& Greaves, J. (2012). Inteligencia emocional 2.0: Estrategias para conocer y aumentar su coeficiente. España: Penguin Random House .

ISSN: 1390-9320, Vol. 4, No.5, octubre 2017 
Fienco V., G., \& Itúrburo S., J. (2011). La Inteligencia Emocional en la Gerencia de Supply Chain. Universidad Politécnica Salesiana Sede Guayaquil, Unidad de Posgrados . Cuenca: Universitaria Abya-Yala.

Folleto del Programa Cóndor aplicados a la CNT EP (2015); Ecuador.

Goleman, D. (1995). Inteligencia Emocional. En D. Goleman, Inteligencia Emocional (M. Santarrita, Trad., pág. 375). Objetiva.

Goleman, D. (1999). La práctica de la inteligencia emocional (primera ed.). (F. Mora, \& D. González Raga, Trads.) Kairós.

Goleman, D., \& Chemiss, C. (2006). Inteligencia emocional en el trabajo (llustrada ed.). (M. Portillo, Trad.) Kairós.

Goleman, D., Goleman, D., Boyatzis, R. E., \& McKee, A. (2002). Lider Resonante Crea Mas. Plaza \& Janes Editories, S.A.

Peña, A. B. (2012). Desarrollo Humano. Madrid, España: Visión Libros.

Troya, M. C. A. (2014). Análisis de la importancia de la inteligencia emocional en la motivación de los trabajadores. Tesis, Universidad Andina Simón Bolívar, Quito.

Urbano, A. (2012). Compartir conocimiento y trabajo colaborativo. Recuperado 22 de agosto de 2016. El individuo dentro de la organización: https://sites.google.com/site/groupccygv/wiki-del-proyecto/1-las-organizacionescomo-generadoras-de-conocimiento-1/2-2-el-individuo-dentro-de-la-organizacion.

Zárate, L., E. M. (s.f.). Inteligencia Emocional y la Actitud de los colaboradores en el ambiente laboral. Tesis, Universidad Rafael Landivar, Facultad de Humanidades, Quetzaltenango. 